\title{
Increasing Colorectal Cancer Burden Among Young US Hispanics: Is It Time to Change Current Screening Guidelines?
}

\author{
Marcia Cruz-Correa
}

Received: 23 April 2013/Accepted: 24 April 2013/Published online: 31 May 2013

(C) Springer Science+Business Media New York 2013

\section{Background and Significance}

The disease burden for colorectal cancer (CRC) varies considerably according to race and ethnicity. A variety of factors, including adherence to CRC screening, are thought to contribute to the racial/ethnic differences in CRC incidence and mortality [1]. In the US, African Americans (A-A) have the highest incidence CRC and the lowest survival rates compared with other racial groups; Hispanics are diagnosed at a later stage and have worse survival compared to with non-Hispanic whites (NHW) [2, 3]. Since Hispanics, compared with NHW and A-A have lower CRC screening rates, they are less likely to be diagnosed at early stages, reducing survival rates [4]. Moreover, since CRC is ranked as one of the top-three causes of cancer-related deaths in US Hispanics, it is a major cause of mortality in this population [5].

Recent epidemiological studies have reported an increase in the incidence of sporadic early onset $(<50$ years old) CRC despite a decrease in the incidence of CRC in older individuals, probably due to routine screening $[6,7]$. Tumors from early onset CRC patients are characteristically microsatellite stable, are located in the distal colon, and have advanced histological features and more advanced staging compared to late-onset tumors, contributing to a higher mortality rate [8-10]. Furthermore, early onset CRC is more often complicated by metastasis either at presentation or during the disease course compared with CRC patients $>50$ years old [10]. The population aged $<50$ thus presents an epidemiologic challenge since they are not covered by current screening guidelines, which currently target individuals $\geq 50$ years old, with

M. Cruz-Correa $(\bowtie)$

University of Puerto Rico Comprehensive Cancer Center,

San Juan, Puerto Rico, USA

e-mail: marcia.cruz1@upr.edu the exception of the A-A population ( $\mathrm{C} 45$ years old) or those individuals with family history of CRC [11].

\section{Integration}

In this issue of Digestive Diseases and Sciences, Jafri et al. [12] examine the temporal trends in the incidence, age at diagnosis, anatomic location, and survival of US Hispanics using population-based data from the Surveillance, Epidemiology, and End Results (SEER) program. The SEER registry program includes all new cancer cases diagnosed in five states and six metropolitan areas, which account for $26-71 \%$ of the racial/ethnic population in the United States. A total of 17,136 Hispanic CRC patients diagnosed between 1993 and 2007 were included in the analysis. Approximately $14 \%$ of all Hispanics were 20-49 years old.

While the annual age-adjusted incidence rates in US Hispanics declined for men and women during 1993-2007, age-specific annual incidence rates among young male and female US Hispanics aged 20-49 increased by $45 \%$ from 3.5 per 100,000 (95\% CI 3.1-3.9) in 1993-1997 to 5.3 per 100,000 (95 \% CI 4.9-5.8) from 2003 to 2007. Significant but less pronounced increases in CRC incidence were also observed in young NHW and African Americans. The striking rise in the incidence of CRC among younger Hispanics was observed in the distal and proximal colon with increases of 50 and $150 \%$, respectively, during the study period. In US Hispanic individuals undergoing screening colonoscopies, over two-thirds of lesions were located proximally [13]. Most strikingly, almost two-thirds of US Hispanics aged 20-49 years were diagnosed with regional $(44 \%)$ or distant spread $(28 \%)$. This finding of advanced CRC staging among young US Hispanics suggests either that colorectal cancer has been present for 
several years (possibly over a decade) or that the adenomacarcinoma sequence is accelerated.

This study presents a comprehensive examination of CRC epidemiology and secular trends using a robust design that highlights the emerging problem of early onset colorectal cancer among US Hispanics. Several potentially modifiable risk factors are highly prevalent among US Hispanics including obesity, diabetes mellitus, and the metabolic syndrome, which significantly increase the risk of CRC by two to threefold. SEER does not collect data on family history of CRC, obesity, diabetes mellitus, metabolic syndrome, or other environmental exposures, limiting our ability to examine the contribution of these risk factors to the increase of CRC incidence among young US Hispanics.

Hispanics trace their origins to Amerindian, European, and African populations, which may contribute genetic and environmental influences that affect the observed CRC incidence trends among young US Hispanics [14]. Jafri and colleagues reported geographical differences in CRC incidence, with higher incidence observed among US Hispanics from New Mexico (predominantly originating from Amerindian-European people) and lower rates in Atlanta (predominantly originating from African-European people). Nevertheless, data regarding the Hispanics tracing their origins to the Caribbean and Central American vs. South American people are not available in SEER, limiting the ability to include this population in the analysis.

\section{Summary and Future Directions}

The manuscript by Jafri et al. [6, 15] adds to the accumulating body of evidence reporting an increase in the incidence rates of CRC among US Hispanics aged $<50$. This increase has been observed despite a decrease in the incidence of CRC in individuals aged $\geq 50$ years, perhaps due to the inclusion of latter population within current screening guidelines recommended by the US Preventive Services Task Force. The paper highlights an increased burden of CRC among young individuals who are excluded from current CRC screening guidelines, which is present in all race/ethnic groups but was significantly higher among US Hispanics. Results from this investigation challenge current screening paradigms and prompt a formal evaluation of the incidence patterns among other Hispanic populations in larger cohorts, including other predominantly Hispanic cancer registries [16] to address if changes to the current CRC screening guidelines should be considered. Additionally, analysis of the cost-effectiveness of CRC screening for Hispanic individuals aged $<50$ is warranted. US Hispanics are the second-largest and the fastest-growing ethnic group in the US, with a population of 42.6 million, accounting for $14 \%$ of the US population. This proportion is predicted to increase by $25 \%$ by the year 2050 . The observed trends for CRC incidence among young US Hispanics will most likely continue affecting larger numbers of US citizens, thus a formal revision of screening guidelines for this ethnic US minority is warranted.

\section{References}

1. Gellad ZF, Provenzale D. Colorectal cancer: national and international perspective on the burden of disease and public health impact. Gastroenterology. 2010;138:2177-2190.

2. Reyes-Ortiz CA, Eschbach K, Zhang DD, Goodwin JS. Neighborhood composition and cancer among Hispanics: tumor stage and size at time of diagnosis. Cancer Epidemiol Biomarkers Prev. 2008;17:2931-2936.

3. Stefanidis D, Pollock BH, Miranda J, et al. Colorectal cancer in Hispanics: a population at risk for earlier onset, advanced disease, and decreased survival. Am J Clin Oncol. 2006;29:123-126.

4. Natale-Pereira A, Marks J, Vega M, Mouzon D, Hudson SV, Salas-Lopez D. Barriers and facilitators for colorectal cancer screening practices in the Latino community: perspectives from community leaders. Cancer Control. 2008;15:157-165.

5. Varela A, Jandorf L, Duhamel K. Understanding factors related to Colorectal Cancer (CRC) screening among urban Hispanics: use of focus group methodology. J Cancer Educ. 2010;25:70-75.

6. Siegel RL, Jemal A, Ward EM. Increase in incidence of colorectal cancer among young men and women in the United States. Cancer Epidemiol Biomarkers Prev. 2009;18:1695-1698.

7. Jafri NS, Gould M, El-Serag HB, Duan Z, Davila JA. Incidence and survival of colorectal cancer among Hispanics in the United States: a population-based study. Dig Dis Sci. 2012. doi:10.1007/ s10620-012-2454-3.

8. Chan TL, Curtis LC, Leung SY, et al. Early onset colorectal cancer with stable microsatellite DNA and near-diploid chromosomes. Oncogene. 2001;20:4871-4876.

9. Boardman LA, Johnson RA, Petersen GM, et al. Higher frequency of diploidy in young-onset microsatellite-stable colorectal cancer. Clin Cancer Res. 2007;13:2323-2328.

10. Chang DT, Pai RK, Rybicki LA, et al. Clinicopathologic and molecular features of sporadic early onset colorectal adenocarcinoma: an adenocarcinoma with frequent signet ring cell differentiation, rectal and sigmoid involvement, and adverse morphologic features. Mod Pathol. 2012;25(8):1128-39.

11. Dominic OG, McGarrity T, Dignan M, Lengerich EJ. American College of Gastroenterology guidelines for colorectal cancer screening 2008. Am J Gastroenterol. 2009;104:2626-2627; author reply 2628-2629.

12. Jafri NS, Gould M, El-Serag HB, Duan Z, Davila JA. Incidence and survival of colorectal cancer among Hispanics in the United States: a population-based study. Dig Dis Sci. 2012. (Epub ahead of print). doi:10.1007/s10620-012-2454-3.

13. Lathroum L, Ramos-Mercado F, Hernandez-Marrero J, Villafana M, Cruz-Correa M. Ethnic and sex disparities in colorectal neoplasia among Hispanic patients undergoing screening colonoscopy. Clin Gastroenterol Hepatol Off Clin Pract J Am Gastroenterol Assoc. 2012;10:997-1001.

14. Salari K, Choudhry S, Tang H, et al. Genetic admixture and asthma-related phenotypes in Mexican American and Puerto Rican asthmatics. Genet Epidemiol. 2005;29:76-86.

15. Meyer JE, Narang T, Schnoll-Sussman FH, Pochapin MB, Christos PJ, Sherr DL. Increasing incidence of rectal cancer in 
patients aged younger than 40 years: an analysis of the surveillance, epidemiology, and end results database. Cancer. 2010;116: 4354-4359.
16. Figueroa-Valles NR, Ortiz-Ortiz KJ, Perez-Rios N, Villanueva-Rosa E, Traverso-Ortiz M, Torres-Cintron CR, Suarez-Ramos T. Cancer in Puerto Rico, 2004-2009. Puerto Rico Central Cancer Registry 2012. 